\title{
Information Technology and Patient Health: An Expanded Analysis of Outcomes, Populations, and Mechanisms
}

\author{
Seth Freedman, Haizhen Lin and Jeffrey Prince*
}

May 2014

\begin{abstract}
This paper examines the effect of hospital adoption of electronic medical records (EMRs) on health outcomes, particularly patient safety indicators (PSIs). We find evidence of a positive impact of EMRs on PSIs via decision support rather than care coordination. Consistent with this mechanism, we find an EMR with decision support is more effective at reducing PSIs for less complicated cases, using several different metrics for complication. These findings indicate the negligible impacts for EMRs found by previous studies focusing on the Medicare population and/or mortality do not apply in all settings.
\end{abstract}

\footnotetext{
${ }^{*}$ Freedman is at the School of Public and Environmental Affairs at Indiana University; Lin and Prince are at the Department of Business Economics and Public Policy in the Kelley School of Business at Indiana University. We thank Leila Agha, Chris Forman, Shane Greenstein, Avi Goldfarb, Jeffrey McCullough, and Amalia Miller for useful discussion and comment, and Noah Hammarlund for excellent research assistance. We also acknowledge the Health Information Management Systems Society (HIMSS) for providing access and assistance to their data. We are responsible for all remaining errors.
} 


\section{Introduction}

The increasing availability and adoption of electronic medical records (EMRs) of various forms has generated substantial optimism concerning possible consequent improvements in productivity, costs, and quality within the healthcare sector (e.g., Hillestad et al., 2005). This optimism has proven substantial enough to even spur the passage of the Health Information Technology for Economic and Clinical Health (HITECH) Act in 2009, which includes financial incentives for adoption. In contrast, the extant literature measuring various impacts of EMR adoption provides little indication of dramatic returns (e.g., Ahga 2012, McCullough et al. 2010, McCullough et al. 2013, Parente \& McCullough 2009). However, the scope of these analyses, particularly with regard to health outcomes, has largely been limited due to data constraints. In particular, previous studies have focused on mortality among Medicare patients as the primary health outcome, and large-scale studies generally have focused only on the Medicare population. The lone large scale study that we are aware of that uses data from non-Medicare patients is Miller and Tucker (2011) who find that the availability of EMRs within a county decreases infant mortality rates. ${ }^{1}$

In this paper, we build and utilize a newly integrated dataset to analyze the effect of EMR adoption on health outcomes for a broader patient population, and for less severe, more common adverse health outcomes known as patient safety indicators (PSIs), detailed below. Our data allow us to examine whether EMR adoption impacts PSIs for the general population, and whether the size of the impact varies according to a patient’s level of “complication,” measured in several different ways. Further, by examining multiple health outcomes (i.e., several different PSIs), multiple types of EMRs, and accounting for differing levels of patient complication, we are able to learn more about potential mechanisms through which EMRs impact health outcomes. In particular, EMRs may impact health outcomes via decision support, information management, and/or care coordination, and these mechanisms, if at work, have different implications according to health outcome, EMR, and patient complication. We can then test

\footnotetext{
${ }^{1}$ Athey and Stern (2002) find information technology linking 911 caller identification to a location database speeds emergency response and reduces short-term mortality and hospital costs.
} 
these implications against what we observe in the data to distinguish the channels through which EMRs have an impact.

Expanding the population and type of health outcome for which we have an empirical assessment of EMRs' effectiveness, along with an improved understanding of the mechanisms by which they operate, can have a significant impact on public policy and subsequent EMR research. It can have a powerful impact on how the U.S. government should be setting policy as pertains to EMR. It will help to better assess the potential social value of incentive programs and whether targeted incentives may be warranted. Further, it can help inform (potential) patients in their choice of care. If benefits for health outcomes from EMRs exist, aware patients, particularly those for whom EMRs might be especially beneficial, can selfselect toward hospitals utilizing these beneficial technologies, improving patient welfare and providing further incentive for non-adopters to adopt sooner.

A significant group of studies has analyzed the effect of EMR adoption on health outcomes, focusing on only the senior population. Within this group of studies, Agha (2012) finds little effect of EMR adoption on patient mortality, medical complication rates, adverse drug events, and readmission rates. Kazley \& Ozcan (2008) find some improvements in process indicators, but overall conclude that the evidence of a relationship between EMRs and quality is limited. McCullough et al. (2010) find some evidence of improvement in quality measures, but temper this finding by concluding that achieving substantive benefits from EMR adoption at a national level may be a lengthy process. McCullough et al. (2013) find evidence of improvement in mortality rates, but only for the most complex cases. Parente \& McCullough (2009) is the paper most similar to ours. They find some improvement in patient safety due to EMR adoption, but conclude there is not enough evidence to draw a strong link between EMR and improvements in patient safety for the Medicare population.

Other studies analyzing the effect of EMR adoption on health outcomes have included the nonsenior population, but have utilized small, focused sets of data, primarily at the hospital level. These studies appear to have more positive findings. For example, Bates et al. (1998) find a significant reduction in serious medication errors due to EMR adoption (computerized physician order entry) in a 
large tertiary care hospital setting. Bates et al. (1999) find a similar improvement in three medical units of a large hospital. Javitt et al. (2008) examine a randomized implementation of decision support tools within an HMO population and find that these tools reduced costs and improved quality. The only large scale study of the non-senior population that we are aware of is Miller and Tucker (2011). They find important effects of EMRs on infant mortality rates, and our study adds to this by examining health effects for a broader subset of the non-elderly population. In addition, our main outcomes of interest are measures of patient safety, instead of mortality. Beyond being a more relevant measure for the non-senior population, patient safety can shed light on the impacts of EMRs on important, but non-deadly, adverse health events.

Prior findings pertaining to costs generally have been negative. For example, Agha (2012) finds no cost savings following EMR adoption, even five years after the fact. Dranove et al. (2012) find that EMRs, on average, generate a slight increase in costs. However, this average effect is a combination of cost reductions for hospitals in "favorable" locations (i.e., due to complementarities) and large cost increases for hospitals in "unfavorable” locations. Sidorov (2006) surveys a range of medical studies on EMRs, arguing that many of these studies suggest costs increased after adoption. In contrast, Wang et al. (2003) argue that cost savings are possible, using a hypothetical cost/benefit analysis within an ambulatory care setting. In addition, Chaudhry et al. (2006) find some efficiency gains in a survey of institution-level data. However, despite these relatively more sanguine findings concerning costs, the majority of the literature suggests cost savings have been small or non-existent.

Our primary data sets include the 2003 through 2010 Health Information and Management Systems Society (HIMSS) Analytics Database and the Nationwide Inpatient Sample (NIS) collected by the Agency for Healthcare Research and Quality’s (AHRQ) Healthcare Cost and Utilization Project (HCUP). To our knowledge, this is the first study to combine data on EMR adoption with a nationally representative sample of hospital discharges and examine the effects of hospital EMR adoption on such a broad patient population. Beyond construction of a novel and integrated dataset, the other primary innovation of this study is the direction in which it takes analysis of the effects of EMR adoption. Prior 
work has consistently analyzed the effects of EMR adoption for very specific subgroups of the population - in particular, the senior population or very small groups of hospitals. Like McCullough et al. (2013), this study calls attention to the real and consequential possibility that EMR adoption may have different impacts across various population subgroups depending on the underlying mechanism.

To test for the effect of EMR adoption on PSIs, we employ a fixed effect approach, exploiting the fact that many hospitals adopted new EMR systems during our time period. We are therefore able to control for fixed differences between adopting and non-adopting hospitals and identify how adopting an EMR changes PSI rates within the adopting hospital. In addition to estimating the overall effect of EMR adoption on PSIs, we estimate the relationship separately according to patient complication (measured by age, complexity, commonality, and severity). Our empirical analysis focuses on two EMR applications, Computerized Physician Order Entry (CPOE) and Physician Documentation, which have experienced large variation in adoption during our study period. Our results suggest that CPOE decreases the occurrence of preventable adverse events as measured by PSIs. This stands in contrast to previous results focusing on mortality as an outcome and suggests EMRs may have important effects on patient outcomes less severe than mortality. We also find that CPOE leads to larger improvements for less complicated cases. Assuming decision support is more likely to be helpful for less complicated cases vis a vis information management and/or care coordination, this finding is suggestive of decision support playing an important role in EMR effectiveness. This notion is further corroborated by the fact that we do not find comparable effects for Physician Documentation, given CPOE is more amenable to decision support while Physician Documentation is more amenable to information management and care coordination.

These findings suggest interesting implications about the effectiveness of electronic medical records in improving health outcomes. First, our findings suggest that they may play a role in improving patient wellbeing by decreasing preventable adverse events. While other research has found little impact on mortality, our results highlight the importance of exploring other health outcomes. Additionally, as discussed below, patient safety indicators have been linked to longer hospital stays and higher hospital charges. Therefore our results may have implications for EMRs role in reducing the cost of care by 
limiting adverse events with additional downstream costs. Second, our results highlight that EMRs have differential effects for population subgroups. In particular, our finding that EMRs are more impactful for less complicated patients suggests that studies of the efficacy of EMRs should take such heterogeneity into account. Furthermore, it suggests that further improvements in how EMRs are used are necessary for them to meaningfully impact the care of more complicated patients. Finally, our findings suggest that the decision support functions of EMRs do lead to improved patient quality, as measured by an outcome these tools are intended to impact: preventable adverse events. We do not find evidence that information management and care coordination play a large role for the broad patient population in our study, suggesting further improvement in interoperability of EMR systems and improved ability to take advantage of large amounts of data provided by EMR systems are necessary to reap their full benefits.

\section{Electronic Medical Records and Health Outcomes}

\subsection{What are EMR Technologies?}

As noted in Dranove et al. (2012), an electronic medical record (EMR) is a "catchall expression used to characterize a wide range of technologies used by hospitals to keep track of utilization, costs, outcomes, and billings.” Some technologies generally classified as EMRs include: Enterprise EMR, Clinical Data Repository (CDR), Clinical Decision Support System (CDSS), Order Entry, Computerized Practitioner Order Entry (CPOE), and Physician Documentation. Wang (2012) considers all six of these technologies in her analysis, and Dranove et al. (2012) consider all but Enterprise EMR.

The functionality of these EMR technologies perhaps is best presented via categorization. Both Dranove et al. (2012) and Wang (2012) break EMR technologies into two broad groups, which can essentially be labeled "basic” and “advanced.” The basic group includes Enterprise EMR, CDR, CDSS, and Order Entry, and the advanced group includes CPOE and Physician Documentation. As Wang (2012) describes, this basic group contains applications that “can be used to store, organize and retrieve patients’ information” and the advanced group presents medical history, recommend drugs, and help 
health care providers make better decisions. Dranove et al. (2012) note that these advanced applications “are more difficult to implement and more difficult to operate successfully due to the need for physician training and involvement."2

We focus our main analysis on CPOE. We make this choice for two reasons. First, its adoption shows the most variation during our data period (details in Section 3). Second and more importantly, CPOE is expected to have direct links to patient safety. As described in McCullough et al. (2013), CPOE allows physicians to directly input orders, potentially reducing miscommunication and errors.

Additionally, rules-based protocols, treatment guidelines, and prescription error checking are often built into CPOE products. ${ }^{3}$ These types of features that provide automatic reminders, check lists, and error checking may be expected to have direct impacts on preventable adverse events that the patient safety indicators we examine are intended to measure.

In addition to CPOE, we also provide some analyses for Physician Documentation, which is the other application that is categorized as an advanced level of EMR. Physician Documentation allows physicians to input information. It also generates diagnostic codes from clinical information. These codes can be used both for billing purposes, but also to enhance communication between practitioners through standard coding (Dranove et al. 2012). Physician Documentation may be expected to reduce adverse events when care is administered by multiple practitioners who must communicate efficiently and coordinate a patient's care.

\subsection{Why Do Hospitals Adopt EMR Technologies?}

The primary reasons cited for hospitals to adopt EMR technologies revolve around healthcare quality and costs. For example, President Obama stated on January 8, 2009 the following: “To improve

\footnotetext{
${ }^{2}$ It is worth noting that Agha (2012) has a slightly different means of characterizing these technologies. Her first group consists of applications whose primary functions are record keeping; and the second being Clinical Decision Support (CDS) whose primary functions are decision support.

${ }^{3}$ CDS application can also provide diagnosis and treatment recommendations based on clinical information. As in McCullough et al. (2013) we do not focus on the CDS application itself due to inconsistent reporting in the HIMSS data.
} 
the quality of our health care while lowering its cost, we will make the immediate investments necessary to ensure that within five years, all of America's medical records are computerized. This will cut waste, eliminate red tape, and reduce the need to repeat expensive medical tests. But it just won't save billions of dollars and thousands of jobs - it will save lives by reducing the deadly but preventable medical errors that pervade our health care system.”

Adoption of EMRs can reduce costs for hospitals by eliminating redundancy, as noted by the President. Further, as noted in Hillestad et al. (2005), EMR adoption can lower costs by reducing drug, radiology, and laboratory usage, reducing clerical staff, reducing nursing time, lowering medical errors, and shortening inpatient lengths of stay. Adoption of EMRs can improve healthcare quality by reducing errors and improving disease prevention and chronic disease management (Hillestad et al., 2005). In addition to these direct benefits to an adopting hospital, as Wang (2012) notes, EMR adoption may generate externalities, meaning its value to one hospital depends on the adoption decisions of other hospitals. Specifically, the value of adopting EMR for a given hospital may increase as a function of the number of other hospitals with EMR, since information transfer becomes easier as more hospitals participate. However, the opposite may be true if EMR adoption attracts more patients, such that ultimately the profits of adoption go down as the number of adopters increases.

In deciding whether to adopt EMR, a hospital must balance the above (potential) benefits against the costs of adopting. The Congressional Budget Office (CBO, 2008) estimates the cost of EMR adoption for a typical urban hospital to range between $\$ 3$ and $\$ 9$ million, along with between $\$ 700,000$ and \$1.35 million per year for maintenance. The costs and benefits of adoption certainly change over time, as well as awareness levels across hospitals and patients. Hence, as we discuss in Section 3, there is significant variation in hospitals' timing of adoption of EMR technologies. This variation is important for us to identify the health effects of these technologies, and our econometric methods are designed to account for potential factors that may concurrently influence EMR adoption and health outcomes, as we discuss in our Methods section. 


\subsection{What are Patient Safety Indicators?}

One innovation of this study is the use of patient safety indicators (PSIs). PSIs comprise a particularly important set of outcomes for the purpose of identifying the effect of EMR adoption. Developed by AHRQ, PSIs are intended to measure preventable in-hospital complications and adverse events. These indicators are well-defined and have gained traction as a health outcome of interest in the general literature on hospital and policy interventions on quality of care (e.g., Iizuka 2013). One advantage of these measures is that they are more variable than other health outcome measures, such as mortality.

PSIs allow us to examine effects of EMR adoption on meaningful health outcomes that may be less severe than mortality. Patient mortality has been commonly referred to as a reliable indicator for quality of care and a patient's outcome. However, being an extreme outcome measure, patient mortality might fail to capture improvement in health outcome resulting from EMR adoption if the patients most likely to be impacted by EMRs are not sick enough to experience mortality. As we discuss below, this might be particularly true of patients most impacted by clinical decision support aspects of EMR technologies.

In addition to representing a significant indicator of patient well-being, PSIs are likely linked to increased healthcare utilization and cost. Zhan and Miller (2003) use the 2000 Nationwide Inpatient Sample to examine how adverse events measured by patient safety indicators impact health care utilization and eventual mortality. They use a multivariable matching estimator to compare length of stay, hospital charges, and in-hospital mortality for patients experiencing an adverse event to observably similar patients within the same hospital not experiencing an adverse event. They find statistically significant differences in these three outcomes for all of the PSIs that we examine in this paper. For example, they find that patients with postoperative pulmonary embolism or deep vein thrombosis spend 5.36 additional days in the hospital, have higher hospital charges by $\$ 21,709$, and have a $6.56 \%$ higher in hospital mortality rate. While these differences may be partially driven by unobserved severity, they 
motivate that PSIs have important implications for downstream health care utilization and costs, and in addition to representing decreased patient well-being, can lead to potential increases in mortality.

As mentioned above, we know of one prior study by Parente \& McCullough (2009) that has analyzed the effect of EMR adoption on PSIs. But their study was limited to a sample of Medicare patients during 1999 and 2002. They also study a different set of EMRs such as nurse chartsand picture archiving and communications systems. The PSIs they utilized were: infection due to medical care, postoperative hemorrhage or hematoma, and postoperative pulmonary embolism or deep vein thrombosis. We look at a broader list of PSIs; however, due to data changes, we do not include infection due to medical care.

We focus our analysis on the following PSIs: postoperative hemorrhage or hematoma, postoperative physiologic and metabolic derangement rate, postoperative respiratory failure rate, and postoperative pulmonary embolism or deep vein thrombosis. In presenting our main results, we aggregate these into a single measure of those experiencing at least one of these four postoperative adverse events. We made our choice of PSIs based on the opinion of clinical experts, following the strategy of Parente \& McCullough (2009). We have chosen PSIs that are most likely to be impacted by the availability of EMRs, in particular those that measure adverse events that can be prevented by checklists and reminders and result from a failure to provide appropriate medication and physical activity (potentially in combination). We exclude from our analysis PSIs that occur with extremely low incidence.

In addition to examining this set of PSIs that are most likely to be directly linked to EMRs, we also examine a number of PSIs that measure adverse events tied more directly to surgical skill and physical accident. We present results of these PSIs as a falsification test. Appendix Table 1 presents definitions of all of the PSI outcomes used in our analysis.

\subsection{Mechanisms and Possible Heterogeneous Effects of EMR Adoption}

Following McCullough et al. (2013), EMRs may be expected to impact health outcomes through three broad channels: clinical decision support, information management, and care coordination. Clinical 
decision support includes a variety of tools that, in conjunction with patient data, can supply the provider with rules-based protocols, treatment guidelines, and error checking. Information management and care coordination are related mechanisms through which EMR technologies can assist providers in monitoring large amounts of clinical data about their patients and in coordinating treatment between multiple providers.

These mechanisms each lead to different predictions about which types of patients may benefit most from EMR adoption. McCullough et al. (2013) suggest that decision support may be most beneficial for moderate to low complexity patients, arguing that "Standard treatment guidelines are rarely implemented for complex combinations of diagnoses.” In other words treatment guidelines, protocols, and reminders, are less beneficial when providers must care for patients with multiple interacting conditions and complexities. Alternatively, information management and care coordination mechanisms are most likely to play a role in high complexity patients, particularly those with conditions that involve synthesizing a large amount of clinical information or many different types of providers. McCullough et al. (2013) find EMRs to have no overall impact on mortality among Medicare patients, but evidence that EMRs improve mortality for the highest severity patients. They further decompose these effects to find the largest benefits for patients with conditions most relevant to information management and care coordination.

While they do not find empirical evidence for the prediction that decision support applications have meaningful health effects in less complex cases, our context may lead to different results since our analysis uses a more diverse patient population. On average, older patients are likely to have greater complexity, therefore leading decision support to be a more important mechanism for younger patient populations. Interestingly, Javitt et al. (2008) does find improvements in quality of care from decision support in an HMO population of patients all under 65 years of age. As discussed below, we begin our analysis by estimating differential effects of EMR adoption by age before exploring additional measures of patient complications and complexity. We then further decompose our estimates by comparing patients with different levels of complications within age groups. 
We might also expect different outcomes to respond differentially to these mechanisms. In particular, patient safety indictors might better capture differences in health outcomes for less complex cases as compared to mortality. Therefore, by examining these less severe, but medically meaningful outcomes, we may be able to better detect the effects of decision support on care quality. Also, as discussed above, the PSIs in our main analysis are especially likely to respond to decision support mechanisms, since protocols and reminders are expected to directly decrease the likelihood of these preventable adverse events.

Finally, these mechanisms also point to potential differential effects by type of EMR technologies. While the primary technology we focus on, CPOE, does not necessarily include decision support tools, it provides a platform by which the physician directly interacts with the IT system to submit orders, and decision support is often incorporated into the CPOE interface. In contrast we expect that the adoption of physician documentation to be more relevant to the information sharing mechanisms through its ability to allow physicians to clearly document and communicate patient information.

\section{Data}

\subsection{Data Construction}

The data we use for this study come from several sources, and to our knowledge, this is the first study using such integrated data. Our first source of data is the Healthcare Information and Management Systems Society (HIMSS) Analytics Database. HIMSS conducts an annual survey of health care providers, including over 3,000 hospitals nationwide with more than 100 beds. The survey collects a wide range of information on more than 100 different health information technology applications, including CPOE and Physician Documentation. For each of these applications we construct variables for whether or not a hospital has a system installed in a given year. ${ }^{4}$ The HIMSS data we have span 2003 to 2010.

\footnotetext{
${ }^{4}$ Following the guidance from HIMSS, we consider an application as installed if its status in the HIMSS data is live and operational, automated, to be replaced, or replaced.
} 
Our second data source is the Nationwide Inpatient Sample (NIS), collected by the Agency for Healthcare Research and Quality’s (AHRQ) Healthcare Cost and Utilization Project (HCUP). Using these data, we are able to build measures of patient safety, and our coverage also spans the years 2003 to 2010. The NIS is a 20-percent, nationally representative, stratified sample of U.S. community hospitals. Since NIS includes the universe of inpatient discharge records from these sampled hospitals, we are able to observe both Medicare and non-Medicare insured patients. For each discharge record, the data set includes information such as diagnosis and procedure codes, admission and discharge status, patient demographics, expected source of payment, length of stay, and hospital charges. The NIS also reports basic hospital characteristics including size, location, ownership type, and number of total discharges.

As noted above, our main outcomes of interest are Patient Safety Indicators (PSIs). We calculate these indicators using a module provided by AHRQ. This module uses information in the discharge record, such as age, diagnosis related groups, diagnosis codes, and procedure codes to identify the subpopulation of patients for whom a particular adverse event is relevant and those who are likely to have experienced the adverse event. For example, for the PSI indicating Postoperative Hemorrhage or Hematoma, the module first identifies patients who have received operations and might be at potential risk, and then it determines which of these patients have experienced a hemorrhage or hematoma.

We supplement the HIMSS and NIS data with American Hospital Associate (AHA) data. The AHA data is used to build a crosswalk between the HIMSS and NIS data. The only external hospital identifier in the HIMSS data is the hospital's Medicare provider numbers. The only external hospital identifier in the NIS data is the hospital's AHA ID number. AHA data contain both identification numbers, thus allowing us to merge the HIMSS and NIS data at the hospital-year level. ${ }^{5}$

Ultimately, our merged sample includes a total of more than 9.1 million observations from 1,912 unique hospitals. While the NIS is not a panel of hospitals, a large fraction of hospitals appear in the data

\footnotetext{
${ }^{5}$ We observe a total of 8,293 observations on 3,858 unique hospitals in the 2003 to 2010 NIS data. Of these, 5,132 observations on 2,377 unique hospitals have AHA identification numbers available in the data. Note that AHA identification numbers are only available for hospitals from a subset of states in the NIS, as some states have not authorized HCUP to release information that would specifically identify hospitals.
} 
in multiple years. For the years 2003 to 2010, we observe 1,223 unique hospitals that appear at least twice, and use these hospitals in our analysis. ${ }^{6}$ This allows us to relate changes in patient safety to changes in EMR adoption within hospitals and over time. Others have used the fact that hospitals appear in the NIS in multiple years to exploit within-hospital changes in other contexts (e.g. Kolstad and Kowalski, 2012).

\subsection{Construction of Key Variables}

Our main analysis focuses on an aggregate PSI which equals 1 if a patient experiences any of the four postoperative adverse events expected to be linked to decision support (PSI9, PSI10, PSI11, and PSI12). We are interested in exploring whether EMR adoption has heterogeneous effects based on a patients' levels of complication, and we use a variety of proxies to measure various dimensions of complications, including case complexity, diagnosis commonality, mortality risk, and functional severity. First we consider the patient age. This variable can serve as a proxy for complication along all four of the above categories. This is because we expect that the non-elderly, on average, present simpler, more common, less risky and less severe cases. An additional advantage of exploring heterogeneity by age is that our results can be directly compared to existing studies which have focused on the elderly population alone through their focus on Medicare data.

Our remaining measures serve to measure one of our complication categories specifically. To measure complexity, we use information on a patient's comorbidities. We define a less complex patient as one having no more than one comorbidity. To measure diagnosis commonality, we utilize diagnosisrelated group (DRG) information. We consider a patient with a DRG code among the top 20 most frequent DRGs for each PSI under study to be more common. To measure risk and severity, we use data available in the NIS severity files that differentiate patients by their mortality risk and their loss of

\footnotetext{
${ }^{6}$ The sample size for each regression described below varies for each PSI, as the number of patients at potential risk for each PSI differ.
} 
function. ${ }^{7}$ Regarding risk type, we define low mortality risk cases as those with a "minor likelihood of dying” and high risk cases as those with "moderate”, "major” or "extreme likelihood of dying.” Regarding severity, we define low severity cases those with "minor loss of function” and high severity cases as those with "moderate," "major" or "extreme loss of function."

\subsection{Summary Statistics}

Table 1 shows the fraction of hospitals in our analysis sample that have adopted CPOE and Physician Documentation by year. CPOE adoption grew from 7\% to 31\% of hospitals from 2003 to 2010. Physician Documentation grew from 18\% to 39\% from 2005 to $2010 .{ }^{8}$ This rapid diffusion provides the key variation we use to identify the effect of EMR adoption on patient safety.

[Table 1 about here]

Table 2 presents summary statistics of our PSI measures. For each PSI used in our analysis, a value of 1 indicates a patient experiencing an adverse event. Note that sample size varies across PSIs because the set of patients that are potentially at risk of each adverse event is different. In Table 2, we present the mean value of each PSI, which indicates the average rate of occurrence among its relevant population. For example, for Agg PSI, the aggregate PSI, we observe an average of $1.81 \%$ with a standard deviation of $13.31 \%$. In our sample, with a total of 9.1 million patients at potential risk, it suggests that about 165,000 patients in the sample had experienced at least one of the adverse events.

[Table 2 about here]

\footnotetext{
${ }^{7}$ The two variables names used are APRDRG_Risk_Mortality and APRDRG_Severity. They are assigned using software developed by 3M Health Information Systems. Their calculation includes the base APR-DRG, the severity of illness subclass, and the risk of mortality subclass within each base APR-DRG. For more information, please refer to the HCUP User Support Website (www.hcup-us.ahrq.gov).

${ }^{8}$ Note that Physician Documentation was first added to HIMSS in 2005. We are able to uncover the status of adoption in 2003 and 2004 for non-adopters (those that did not adopt between 2005 and 2011) and late-adopters (those that adopted between 2005 and 2011). For those that we observe adoption in 2005, we are not able to tell the year of adoption so we do not fill in these missing values.
} 
Table 3 provides summary statistics of the aggregate PSI by patient heterogeneity. Not surprisingly, we find that the rate of occurrence of adverse events is lower for less "complicated” patients. For example, nonelderly patients have the mean of $1.49 \%$ while elderly patients have a mean of $2.21 \%$. Similarly, the mean is $0.53 \%$ for low-risk patients but as high as $4.02 \%$ for high-risk ones. However, PSIs are still meaningful health outcomes for less complicated patients as defined by most of our proxies, as the present of less complicated patients experiencing a PSI are along the same order of magnitude as those with more complicated cases when we separate by age, complexity, and commonality.

[Table 3 about here]

\section{Empirical Model}

Our general empirical strategy for testing the impact of EMR adoption on patient safety outcomes is to relate within-hospital changes in patient safety over time to within-hospital changes in the availability of EMRs. Hospitals that do and do not have EMRs at a point in time may be very different from each other. Therefore we exploit over time variation in EMR adoption. The key identifying assumption is that trends in the prevalence of PSIs are not correlated with unobserved adoption trends. In other words, our empirical strategy hinges on the idea that when a hospital adopts EMRs there are no concurrent events, left unaddressed by our controls, that would have an impact on patient safety. If this assumption is satisfied, we can attribute changes in patient safety to EMR adoption. McCullough et al. (2013) and Agha (2012) provide extensive evidence that EMR adoption is unlikely to be correlated with pre-existing trends in patient outcomes or severity in the Medicare context. In results shown below, we also confirm that these findings apply to the data used in this paper.

Our baseline empirical specification follows a linear probability model, with hospital and time fixed effects: 


$$
P S I_{i h t}=\beta_{0}+\delta_{t}+\alpha_{h}+Z_{i h t} \beta_{1}+E M R_{h t} \beta_{2}+E M R_{h t \_} X_{i h t} \beta_{3}+X_{i h t} \beta_{4}+X_{i h t} * I_{-} \text {year }+\varepsilon_{i h t}
$$

Let $P S I i_{h t}$ represent the occurrence of an adverse event for patient $i$ in hospital $h$ during year $t . \alpha_{h}$ is a set of hospital fixed effects, $\delta_{t}$ is a set of year fixed effects, and $E M R_{h t}$ is a dummy variable for the presence of an EMR system installed in hospital $h$ at year $t . Z_{i h t}$ stands for a set of patient-level control variables, including age, age squared, gender, race dummies, payment dummies, risk dummies, severity dummies, and 27 different comorbidity dummies as defined by AHRQ. ${ }^{9} X_{i h t}$ is one of the following dummies to represent patient heterogeneity: non-elderly, non-complex, common DRG, low-mortality risk, and nonsevere case. We interact each dummy variable $\left(X_{i h t}\right)$ with the $E M R_{h t}$ dummy to allow the effect of EMR to differ on the basis of patient heterogeneity. We also include a group specific year fixed effects $\left(X_{i h t} *\right.$ I_year) to allow outcomes to differ arbitrarily between each group over time.

We are particularly interested in the estimates of $\beta_{2}$, which measures the overall effect of EMR adoption, and $\beta_{3}$, which measures whether the effect varies by patient heterogeneity. $\beta_{4}$ measures whether patient safety indicators vary based on patient heterogeneity. The estimation of the model uses individual discharge weighting and standard errors are clustered at the hospital level.

We provide separate analyses for CPOE and Physician Documentation. Note that the results of the effect of each application do not change if we analyze both EMRs jointly. As indicated in Section 2, there is reason to believe CPOE and physician documentation may have differing impacts on health outcomes, since the former has more capability toward clinical decision support and the latter toward care coordination.

In addition to the baseline model, we extend our model in a number of ways. First, we explore how the impact of EMR adoption on patient safety differs by time since adoption. For this analysis we replace the EMR dummy with three different dummies indicating the first, second, or third or more year of adoption. This specification allows for the fact that it may take some time to fully and optimally

\footnotetext{
${ }^{9}$ All of our results hold if we include some hospital level controls such as hospital bed size, urban vs. rural location, and ownership type.
} 
incorporate EMR usage into practice patterns. Coefficient estimates from this specification reveal how this process evolves from the first year of adoption through later years. This extended model also mitigates a main limitation of our main specification. Because the NIS is not a true panel, our basic model treats hospitals who have adopted EMRs between observation years the same, regardless of which year they actually adopted the technology. By utilizing the HIMSS data to calculate the number of years an application has been installed, we can more precisely differentiate the relationship between actual adoption year and changes in patient safety. ${ }^{10}$

Second, we further explore heterogeneity by examining a three-way interaction of EMR application, non-elderly and non-complicated case. Our baseline model differentiates patients along a single dimension of heterogeneity. This extended model allows for an additional interaction between age and level of complication to test if the effect of EMRs differs by complication within age groups.

\section{Results}

\subsection{Main Results}

We first report our main results for CPOE in Table 4. We find an overall negative but statistically insignificant effect of CPOE on the probability of experiencing an adverse event. Allowing for heterogeneous effects by patient age (column (1)), we find that CPOE has a larger effect on non-elderly patients, suggesting that non-elderly patients are likely to benefit more from CPOE adoption. We see consistent patterns of results when examining heterogeneous effects along other dimensions. For example, we find that complicated cases are not affected by CPOE. However, uncomplicated cases do observe a large decrease in occurrence of adverse events. To be more specific, the adoption of CPOE is associated with a $12 \%((0.18-0.016) / 1.34)$ drop in the probability of experiencing at least one postoperative adverse events for cases with less than one comorbidity (non-complex). Regarding commonality of DRGs, we find no significant effect of CPOE on patients with less common DRGs. However, patients that are

\footnotetext{
${ }^{10}$ Note that the sample size for this set of regressions is smaller as for those hospitals who have adopted EMRs prior to 2003, we are not able to track down the number of years since adoption.
} 
diagnosed with more common DRGs experience a $20 \%((0.14+0.033) / 0.86)$ decrease in the probability of an adverse event after CPOE adoption. Similarly we find largely lowered rate of adverse events for patients with low morality risk (a 43\% decrease in occurrence rate) and low severity (a 135\% decrease).

\section{[Table 4 about here]}

The pattern of estimates observed in Table 4 suggests that it is important to allow for heterogeneous effects when examining the effect of CPOE adoption. These results are also consistent with our prior that CPOE is more likely to improve patient outcomes for less complicated cases through the clinical decision support channel.

We report the same set of results for Physician Documentation in Table 5. We find less pronounced effects for Physician Documentation, and the result is only statically significant for low risk mortality patients. The lack of effect for Physician Documentation seems to be consistent with the fact that Physician Documentation usually records patient information for tracking and billing purposes. For the remainder of the paper, we will focus our analysis on CPOE, since it is the EMR, of the two, that appears to display a notable impact on PSIs. ${ }^{11}$

[Table 5 about here]

\subsection{Endogeneity Concerns}

The fact that hospitals actively decide to adopt CPOE or any type of EMR application poses concerns about the potential bias caused by endogeneity. Our hospital fixed effects account for the fact that adopting and non-adopting hospitals may differ in terms of baseline characteristics, and thus our key identifying assumption is that hospitals that adopt during our sample period do not exhibit differential trends in unobserved factors that might impact changes in PSI prevalence over time. For example, it

\footnotetext{
${ }^{11}$ The corresponding results for Physician Documentation are available upon request.
} 
might be the case that adopting hospitals are those that have been implementing other quality initiatives to improve quality of care. If this is the case, our identification would be contaminated, as we could not differentiate whether the estimated improvement in patient outcome is driven by CPOE or by other quality initiatives. It might also be the case that patient outcomes differ along less and more complicated patient groups prior to the adoption of CPOE. If so, it would be misleading to interpret our results as the causal effect of the adoption of CPOE.

To address the above endogeneity concerns, we adopt two strategies. First, we run our model on a set of PSIs that are not likely to be affected by EMRs because they are mostly the result of physician skill or physical accident. For this group, we should not find any significant effect because otherwise, our results might be driven by other quality initiatives that have been implemented simultaneously with EMR adoption. These results are reported in Table 6 for PSI6, PSI7, PSI8 and PSI15. ${ }^{12}$ For each choice of PSI, we find very small and no statistically significant effects of CPOE on those outcomes, regardless of the type of the patients. While some of the interaction terms are statistically significant, the overall effect of CPOE on each PSI for the less complicated groups are not statistically significant when considering the combination of the main effects and the interaction effects. It is not likely that quality initiatives would work exactly like EMRs (namely they are related to our affected PSIs and not related to our unaffected PSIs). Passing these falsification tests suggests that our results are not likely driven by other quality initiatives by the adopting hospitals.

[Table 6 about here]

Another strategy that we use is to estimate a model which includes adopting lead dummies. We define lead12 as being one or two years prior to adoption and lead34 as being three or four years prior to adoption. The coefficients in front of these two dummies would capture whether there is any differential

\footnotetext{
${ }^{12}$ In Table 6, the sample is restricted to the population at potential risk of our affected PSIs (PSI9 to PSI12). One advantage of using this sample is that the results of the falsification tests could be directly compared to our main results. We have also expanded the sample to all relevant patient population and have found largely similar results.
} 
trend in PSI prevalence between adopting and non-adopting hospitals in the years prior to the adoption actually occurring. Column 0 shows that when we consider all patients together, there were no changes in PSI prevalence prior to CPOE adoption. In the subsequent columns we find no statistically significant differential pre-trends for either complicated or uncomplicated cases, regardless of our measure of complication. Results from the F-test all fail to reject that there is any significant effect of these lead dummies on less-complicate cases when considering all four years prior to adoption together. Similar to our main findings, the contemporaneous effects suggest that less complicated cases see improvements in PSI prevalence after CPOE is adopted.

\section{[Table 7 about here]}

\subsection{Timing of Effect}

We also explore heterogeneity by time of EMR adoption. It could be the case that adoption of a new EMR system may not immediately improve health outcomes. Healthcare providers, such as physicians, nurses, and other staff, must be trained to use new systems. It may take additional time for providers to learn how to use the new systems to optimally impact patient's health. ${ }^{13}$ In this sense, we might see some delay in observing quality improvement.

Table 8 presents estimates separating the effect of adoption by the number of years CPOE has been put in use. While many of the coefficient estimates themselves are not statistically significant, when we combine the main effects and the low complication interactions, we find CPOE has a consistent impact on safety over time for less complicated patients. F-statistics for these combined effects are presented at the bottom of the table. For example, CPOE decreases non-complex patients' probability of experiencing at least one postoperative adverse event by 0.203 (15.1\%) and 0.197 (14.7\%) percentage points in years one and two, respectively. Having CPOE installed for three or more years lowers the

\footnotetext{
${ }^{13}$ Dranove et al. (2012) find that cost savings from EMRs in IT-intensive do not occur immediately and instead materialize 3 years after a system is put in place.
} 
chance of patients experiencing at least one adverse postoperative event by 0.222 (16.6\%) percentage points. These results suggest that the effect of CPOE takes place beginning the first year of use and the effect persists over time.

\section{[Table 8 about here]}

\subsection{Additional Levels of Heterogeneity}

In addition to exploring differential effects of CPOE along various dimensions of patient complication, we also estimate an extended model to allow the effect to vary by age group (elderly vs non-elderly) and case complication. These results are reported in Table 9. The first column replicates previous results of the heterogeneous effect by age group. In column 2, we also include an interaction between CPOE and a dummy for a non-complex case, and a three way interactions between CPOE, nonelderly and non-complex case. The results suggest that the largest decrease in occurrence of adverse events is realized for non-elderly, non-complex cases. In the remaining columns, we also explore commonality of DRG (column 3), mortality risk (column 4), and severity (column 5). We find consistent results that CPOE has the largest impact for non-elderly and non-complicated cases. In addition, we also see some improvement in patient outcomes for elderly but non-complicated cases. By introducing two dimensions of complication, we find the largest effects for patients in the least complicated category when both dimensions are combined. These results provide additional evidence the CPOE is likely to impact patient outcome through the channels that would have the largest impact on the least complicated cases, further suggesting clinical decision support as the most likely mechanism.

\section{[Table 9 about here]}

\subsection{Two Alternative Outcomes}

In addition to PSIs, we also present results using two alternative outcomes: inpatient mortality and length of stay. Mortality has been commonly used in the existing studies examining the impact of EMRs. Previously, we argued one concern about using mortality is that it is considered as an extreme 
outcome, so it might fail to capture improvement in patient outcomes that are not directly tied to patient death. In our data, we observe when a patient dies during their hospital stay, although the results have to be taken cautiously as inpatient mortality does not capture death that occurs during a readmission or outside of the hospital. These results are reported in Table 10. Consistent with previous findings, we find no effect of CPOE on inpatient mortality, regardless of patient characteristics. ${ }^{14}$

\section{[Table 10 about here]}

The other outcome we examine is length of stay (logarithm), and the results are also reported in Table $10 .{ }^{15}$ Interestingly, we find a large decrease in length of stay (LOS) for non-complicated cases, consistent with our main findings that CPOE lowers the probability of adverse events, which themselves are likely to increase length of stay, for those patients. For example, we find that CPOE lowers patient LOS by $1.2 \%$ for low mortality risk patients and $2.10 \%$ for low severity patients. We do not find statistically significant effects for complicated cases, again consistent with our findings for PSIs.

\section{Conclusion}

By combining Healthcare Information and Management Systems Society Analytics Database with the National Inpatient Sample, we test how adoption of advanced electronic medical records (CPOE and physician documentation) affect the incidence of patient safety indicators, and whether this effect differs across case complication. We find that CPOE decreases the prevalence of patient safety indicators that are likely to be amenable to decision support functions, particularly for less complicated patients. We find little impact of physician documentation on these quality measures. Taken together, the fact that we

\footnotetext{
${ }^{14}$ We also estimate an extended model to allow three-way interaction terms for CPOE, non-elderly and dummies for complication. We find consistent results that there is no effect of CPOE on patient mortality.

${ }^{15}$ In addition to providing evidence using an alternative outcome measure, length of stay has the advantage of being a continuous variable. Finding consistent results reassured us of using linear probability model for modeling our discrete dependent variables PSIs.
} 
find the most consistent benefits of EMR adoption when examining a technology with decision support features, outcomes likely to be sensitive to decision support, and patient populations for whom decision support is likely to be most beneficial, our results point to decision support as the most likely mechanism driving our results.

The findings have important implications concerning the impact of EMR adoption on health outcomes. Our results are in contrast to previous large scale studies that focus on Medicare populations, and suggest that younger populations may in fact be currently receiving larger benefits from EMR adoption. While McCullough et al. (2013) find evidence of improved mortality for high severity Medicare patients with diagnoses specifically necessitating information management and care coordination, our results suggest that the impact of these mechanisms is currently limited to a relatively small group of patients. Our findings contribute to the discussion of how to push EMR technologies forward by shedding light on which mechanisms are currently leading to health improvements. While we find that decision support tools are leading to improved outcomes, further research is warranted to understand how providers can better make use of information management and care coordination aspects of EMRs. 


\section{REFERENCES}

Agha, L. 2012. The Effects of Health Information Technology on the Costs and Quality of Medical Care. Working paper, Massachusetts Institute of Technology.

Athey, S and Stern, S. 2002. The Impact of Information Technology on Emergency Health Care Outcomes. Rand Journal of Economics 33: 399-432.

Bates, D., Leape, L., Cullen, D., Laird, N., Petersen, L., Teich, J., Burdick, E., Hickey, M., Kleefield, S., Shea, B., Vliet, M., and Seger, D. 1998. Effect of Computerized Physician Order Entry and a Team Intervention on Prevention of Serious Medication Errors. Journal of the American Medical Association 280: 1311-1316.

Bates, D., Teich, J., Lee, J., Seger, D., Kuperman, G., Ma’Luf, N., Boyle, D., and Leape, L. 1999. The Impact of Computerized Physician Order Entry on Medication Error Prevention. Journal of the American Medical Informatics Association 6: 313-321.

Cameron, A and Trivedi, P. 2005 Microeconometrics: Methods and Applications. $1^{\text {st }}$ Ed., New York: Cambridge University Press.

Chaudhry, B., Wang, J., Wu, S., Maglione, M., Mojlca, W., Roth, E., Morton, S. and Shekelle, P. 2006. Systematic Review: Impact of Health Information Technology on Quality, Efficiency, and Costs of Medical Care. Annals of Internal Medicine 144: 742-752.

Congressional Budget Office. 2008. Evidences on the Costs and Benefits of Healthcare Information Technology. http://www.cbo.gov/ftpdocs/91xx/doc9168/maintext.3.1.shtml. Accessed on January 16, 2013.

Dranove, D., Forman, C., Goldfarb, A., and Greenstein, S. 2012. The Trillion Dollar Conundrum: Complementarities and Health Information Technology. NBER Working paper.

Hillestad, R., Bigelow, J., Bower, A., Girosi, F., Meili, R., Scoville, R., and Taylor, R. 2005. Can Electronic Medical Record Systems Transform Health Care? Potential Health Benefits, Savings, and Costs. Health Affairs 24: 1103-1117.

Iizuka, T. 2013. Does Higher Malpractice Pressure Deter Medical Errors? Journal of Law and Economics 56: $161-188$.

Javitt, J. Rebitzer, J. and Reisman, L. 2008. Information Technology and Medical Missteps: Evidence from a Randomized Trial, Journal of Health Economics 27(23):585-602.

Kazley, A. and Ozcan, Y. 2008. Do Hospitals with Electronic Medical Records (EMRs) Provide Higher Quality Care? An Examination of Three Clinical Conditions. Medical Care Research and Review 65: 496-513.

Kolstad, J. and Kowalski, A. 2012. The Impact of Health Care Reform on Hospital and Preventative Care: Evidence from Massachusetts. Journal of Public Economics 96: 909-929. 
McCullough, J., Casey, M., Moscovice, I., and Prasad, S. 2010. The Effect of Health Information Technology on Quality in U.S. Hospitals. Health Affairs 29: 647-654.

McCullough, J., Parente, S., and Town, R. 2013. Health Information Technology and Patient Outcomes: The Role of Organizational and Informational Complementarities. NBER working paper.

Miller, A. and Tucker, C. 2011. Can Health Information Technology Save Babies? Journal of Political Economy 119: 289-324.

Mold, J., Fryer, G., and Roberts, M. 2004. When Do Older Patients Change Primary Care Physicians? Journal of the American Board of Family Medicine 17: 453-460.

Parente, S. and McCullough, J. 2009. Health Information Technology and Patient Safety: Evidence from Panel Data. Health Affairs 28: 357-360.

Sidorov, J. 2006. It Ain't Necessarily So: The Electronic Health Record and the Unlikely Prospect of Reducing Health Care Costs. Health Affairs 25: 1079-1085.

Wang, S., Bardon, C., Kittler, A., and Sussman, A. 2003. A Cost-Benefit Analysis of Electronic Medical Records in Primary Care. The American Journal of Medicine 114: 397-403.

Wang, Y. 2012. Cooperation and Competition: The Multilevel Adoption of Electronic Medical Records in U.S. Hospitals. Working paper, Boston University.

Zhan, C. and Miller, M. 2003. Excess Length of Stay, Charges, and Mortality Attributable to Medical Injuries During Hospitalization. Journal of the American Medical Association 290: 1868-1874. 
Table 1: EMR adoption by year

\begin{tabular}{ccc}
\hline Year & CPOE & $\begin{array}{c}\text { Physician } \\
\text { Documentation }\end{array}$ \\
\hline 2003 & 0.070 & - \\
2004 & 0.090 & - \\
2005 & 0.165 & 0.182 \\
2006 & 0.175 & 0.211 \\
2007 & 0.198 & 0.234 \\
2008 & 0.268 & 0.288 \\
2009 & 0.304 & 0.391 \\
2010 & 0.313 & 0.386 \\
\hline
\end{tabular}

Notes: This table lists the fraction of hospitals with CPOE and Physician Documentation installed by year. The sample includes all hospitals for which we have at least two observations in the merged HIMSS and NIS data. 
Table 2: Summary Statistics of Patient Safety Indicators

\begin{tabular}{|c|c|c|c|c|c|c|c|c|c|}
\hline & \multicolumn{5}{|c|}{ Postoperative PSIs } & \multicolumn{4}{|c|}{ Control PSIs } \\
\hline & PSI 9 & PSI 10 & PSI 11 & PSI 12 & Agg PSI & PSI 6 & PSI 7 & PSI 8 & PSI 15 \\
\hline Mean (\%) & 0.27 & 0.12 & 1.04 & 1.15 & 1.81 & 0.08 & 0.27 & 0.02 & 1.04 \\
\hline S. D. (\%) & 5.19 & 3.51 & 10.17 & 10.65 & 13.31 & 2.81 & 5.14 & 1.43 & 10.15 \\
\hline N(Million) & 9.06 & 4.24 & 3.38 & 9.08 & 9.10 & 8.18 & 5.57 & 5.86 & 8.51 \\
\hline
\end{tabular}

Note: This table presents summary statistics of each PSI measure. The unit of observation is at the patient level.

Table 3: Summary Statistics for Aggregate PSI

\begin{tabular}{|c|c|c|c|c|c|c|c|c|c|c|}
\hline \multirow[t]{2}{*}{ Case Complication } & \multicolumn{2}{|c|}{ non-elderly } & \multicolumn{2}{|c|}{ non-complex } & \multicolumn{2}{|c|}{ common DRG } & \multicolumn{2}{|c|}{ low mortality } & \multicolumn{2}{|c|}{ low severity } \\
\hline & Yes & No & Yes & No & Yes & No & Yes & No & Yes & No \\
\hline Mean (\%) & 1.49 & 2.21 & 1.34 & 2.36 & 0.86 & 2.28 & 0.53 & 4.02 & 0.22 & 2.82 \\
\hline S. D. (\%) & 12.13 & 14.72 & 11.49 & 15.17 & 9.23 & 14.92 & 7.23 & 19.65 & 4.66 & 16.55 \\
\hline N(Million) & 5.19 & 3.91 & 4.94 & 4.16 & 3.04 & 6.05 & 5.72 & 3.29 & 3.52 & 5.49 \\
\hline
\end{tabular}


Table 4: Effect of CPOE on Patient Safety

\begin{tabular}{lcccccc}
\hline & $\mathbf{( 0 )}$ & $\mathbf{( 1 )}$ & $\mathbf{( 2 )}$ & $\mathbf{( 3 )}$ & $\mathbf{( 4 )}$ & $\mathbf{( 5 )}$ \\
\hline CPOE & -.080 & -.036 & .016 & -.033 & $.174^{*}$ & .055 \\
& $(.080)$ & $(.082)$ & $(.077)$ & $(.084)$ & $(.097)$ & $(.080)$ \\
CPOE_X & & $-.077^{*}$ & $-.180^{* * *}$ & $-.140^{* * *}$ & $-.402^{* * *}$ & $-.352^{* * *}$ \\
& & $(.041)$ & $(.043)$ & $(.045)$ & $(.099)$ & $(.079)$ \\
CPOE+CPOE_X=0 & & 1.84 & $3.47^{*}$ & $4.75^{* *}$ & $6.32^{* *}$ & $8.70^{* * *}$ \\
& & & & & \\
$\mathrm{X}=$ & & Non- & Non- & Common- & Low- & Non-Severe \\
\hline
\end{tabular}

Note: Coefficient estimates are from separate fixed effect linear probability regressions of the aggregate PSIs on dummies indicating adoption of CPOE and its interaction with different measures for patient complication $(X)$. All regressions control for hospital fixed effects, year fixed effects, patient characteristics, $X$, a group specific year fixed effects $\left(X * I_{-}\right.$year $)$. All standard errors are clustered at the hospital level.

$* * *-\mathrm{p}<.01, * *-\mathrm{p}<.05, *-\mathrm{p}<.10$

Table 5: Effect of Physician Documentation on Patient Safety

\begin{tabular}{lcccccc}
\hline & $\mathbf{( 0 )}$ & $\mathbf{( 1 )}$ & $\mathbf{( 2 )}$ & $\mathbf{( 3 )}$ & $\mathbf{( 4 )}$ & $\mathbf{( 5 )}$ \\
\hline PhysDoc & -.002 & .001 & .015 & -.033 & .118 & .042 \\
& $(.032)$ & $(.041)$ & $(.041)$ & $(.037)$ & $(.073)$ & $(.046)$ \\
PhysDoc_X & & -.011 & -.069 & -.054 & $-.200^{* *}$ & $-.122^{*}$ \\
& & $(.040)$ & $(.045)$ & $(.043)$ & $(.095)$ & $(.074)$ \\
PhysDoc+PhysDoc_X=0 & & 0.08 & 0.87 & 0.95 & $3.69 * *$ & 2.43 \\
& & & & & & \\
X= & & Non- & Non- & Common- & Low- & Non- \\
& & Elderly & Complex & Drg & Mortality & Severe \\
\hline
\end{tabular}

Note: Coefficient estimates are from separate fixed effect linear probability regressions of the aggregate PSIs on dummies indicating adoption of Physician Documentation and its interaction with different measures for patient complication $(X)$. All regressions control for hospital fixed effects, year fixed effects, patient characteristics, $X$, a group specific year fixed effects $\left(X * I_{-}\right.$year $)$. All standard errors are clustered at the hospital level.

$* * *-\mathrm{p}<.01, * *-\mathrm{p}<.05, *-\mathrm{p}<.10$ 
Table 6: Falsification Test on Non-affected PSIs

\begin{tabular}{|c|c|c|c|c|c|c|}
\hline & (0) & (1) & (2) & (3) & (4) & (5) \\
\hline \multicolumn{7}{|l|}{ Panel: PS6 } \\
\hline \multirow[t]{2}{*}{ CPOE } & .003 & .001 & 0.001 & .003 & .013 & .008 \\
\hline & $(.005)$ & $(.006)$ & $(0.006)$ & $(.005)$ & $(.008)$ & $(.006)$ \\
\hline \multirow[t]{2}{*}{ CPOE_X } & & .003 & 0.002 & -.004 & $-.016^{*}$ & $-.012^{* *}$ \\
\hline & & $(.005)$ & $(.005)$ & $(.005)$ & $(.009)$ & $(.006)$ \\
\hline $\mathrm{CPOE}+\mathrm{CPOE} \_\mathrm{X}=0$ & & 0.68 & 0.60 & 0.04 & 0.32 & 0.88 \\
\hline \multicolumn{7}{|l|}{ Panel: PS7 } \\
\hline \multirow[t]{2}{*}{ CPOE } & -.005 & -.004 & .010 & .000 & .005 & .002 \\
\hline & $(.015)$ & $(.013)$ & $(.017)$ & $(.016)$ & $(.027)$ & $(.017)$ \\
\hline \multirow[t]{2}{*}{ CPOE_X } & & -.002 & $-.028 * *$ & -.011 & -.015 & .018 \\
\hline & & $(.013)$ & $(.011)$ & $(.013)$ & $(.049)$ & $(.017)$ \\
\hline $\mathrm{CPOE}+\mathrm{CPOE} \_\mathrm{X}=0$ & & 0.13 & 1.29 & 0.64 & 0.49 & 1.08 \\
\hline \multicolumn{7}{|l|}{ Panel: PS8 } \\
\hline \multirow[t]{2}{*}{ CPOE } & -.002 & .000 & .000 & -.002 & -.003 & -.003 \\
\hline & $(-.003)$ & $(.004)$ & $(.003)$ & $(.003)$ & $(.005)$ & $(.003)$ \\
\hline \multirow[t]{2}{*}{ CPOE_X } & & -.003 & $-.005^{*}$ & .000 & .001 & .002 \\
\hline & & $(.003)$ & $(.003)$ & $(.003)$ & $(.004)$ & $(.003)$ \\
\hline CPOE+CPOE_X=0 & & 1.55 & $2.79 *$ & 0.45 & 0.59 & 0.15 \\
\hline \multicolumn{7}{|l|}{ Panel: PS15 } \\
\hline \multirow[t]{2}{*}{ CPOE } & -.014 & .017 & .012 & -.014 & .014 & .008 \\
\hline & $(.029)$ & $(.031)$ & $(.022)$ & $(.031)$ & $(.036)$ & $(.032)$ \\
\hline \multirow[t]{2}{*}{ CPOE_X } & & $-.054 * *$ & $-.050 * *$ & -.020 & -.045 & $-.058 * *$ \\
\hline & & $(.024)$ & $(.022)$ & $(.037)$ & $(.051)$ & $(.028)$ \\
\hline $\mathrm{CPOE}+\mathrm{CPOE} \_\mathrm{X}=0$ & & 1.39 & 1.38 & 0.77 & 0.99 & 2.44 \\
\hline$X=$ & & $\begin{array}{c}\text { Non- } \\
\text { Elderly }\end{array}$ & $\begin{array}{c}\text { Non- } \\
\text { Complex }\end{array}$ & $\begin{array}{l}\text { Common- } \\
\text { Drg }\end{array}$ & $\begin{array}{c}\text { Low- } \\
\text { Mortality }\end{array}$ & $\begin{array}{l}\text { Non- } \\
\text { Severe }\end{array}$ \\
\hline
\end{tabular}

Note: Coefficient estimates are from separate fixed effect linear probability regressions of a set of non-affected PSIs on dummies indicating adoption of CPOE and its interaction with different measures for patient complication (X). All regressions control for hospital fixed effects, year fixed effects, patient characteristics, X, a group specific year fixed effects (X*I_year). All standard errors are clustered at the hospital level.

$* * *-\mathrm{p}<.01, * *-\mathrm{p}<.05, *-\mathrm{p}<.10$ 
Table 7: Lead Effect of Adoption

\begin{tabular}{lcccccc}
\hline & $\mathbf{( 0 )}$ & $\mathbf{( 1 )}$ & $\mathbf{( 2 )}$ & $\mathbf{( 3 )}$ & $\mathbf{( 4 )}$ & $\mathbf{( 5 )}$ \\
\hline CPOE & -.085 & -.040 & .008 & -.035 & $.188^{*}$ & .056 \\
& $(.093)$ & $(.096)$ & $(.091)$ & $(.098)$ & $(.111)$ & $(.095)$ \\
Lead12 & -.023 & -.015 & -.009 & -.183 & .042 & -.002 \\
& $(.048)$ & $(.065)$ & $(.061)$ & $(.055)$ & $(.115)$ & $(.076)$ \\
Lead34 & .013 & .021 & .021 & .037 & .042 & .059 \\
& $(.065)$ & $(.084)$ & $(.080)$ & $(.070)$ & $(.115)$ & $(.077)$ \\
CPOE_X & & -.081 & $-.178^{* * *}$ & $-.147^{* * *}$ & $-.435^{* * *}$ & $-.371^{* * *}$ \\
& & $(.041)$ & $(.044)$ & $(.047)$ & $(.101)$ & $(.078)$ \\
Lead12_X & & -.016 & -.028 & -.006 & -.101 & -.055 \\
& & $. .064)$ & $(.053)$ & $(.041)$ & $(.149)$ & $(.122)$ \\
Lead34_X & & -.015 & .063 & -.066 & -.191 & -.110 \\
& & $. .055)$ & $(.048)$ & $(.057)$ & $(.128)$ & $(.104)$ \\
CPOE+CPOE_X=0 & & & & & & \\
lead12+lead12_X=0 & & 1.63 & $2.89 *$ & $4.10^{* *}$ & $6.00^{* *}$ & $8.55^{* * *}$ \\
lead34+lead34_X=0 & & 0.36 & 0.53 & & & \\
X= & & 0.01 & 0.39 & 0.17 & 0.84 & 0.54 \\
& & Non- & Non- & Common- & Low- & Non- \\
& & Elderly & Complex & Drg & Mortality & Severe \\
\hline
\end{tabular}

Note: Coefficient estimates are from separate fixed effect linear probability

regressions of the aggregate PSIs on dummies indicating adoption of CPOE and its interaction with different measures for patient complication $(\mathrm{X})$, along with dummies variables for 1 or 2 years before adoption (Lead12), and 3 or 4 years before adoption (Lead34), and their interaction terms with X . All regressions control for hospital fixed effects, year fixed effects, patient characteristics, $X$, a group specific year fixed effects (X*I_year). All standard errors are clustered at the hospital level.

$* * *-\mathrm{p}<.01, * *-\mathrm{p}<.05, *-\mathrm{p}<.10$ 
Table 8: Differential Effects by Years since Adoption

\begin{tabular}{|c|c|c|c|c|c|c|}
\hline & (1) & (2) & (3) & (4) & (5) & (6) \\
\hline \multicolumn{7}{|l|}{ CPOE } \\
\hline \multirow[t]{2}{*}{ Year 1} & -.146 & -.059 & -.077 & -.100 & .086 & -.035 \\
\hline & $(.099)$ & $(.097)$ & $(-.085)$ & $(.104)$ & $(.114)$ & $(.095)$ \\
\hline \multirow[t]{2}{*}{ Year 2} & $-.149 * *$ & -0.078 & -0.100 & -.132 & .055 & -.008 \\
\hline & $(.074)$ & $(.084)$ & $(.079)$ & $(.080)$ & $(.128)$ & $(.093)$ \\
\hline \multirow[t]{2}{*}{ Year 3} & -.148 & -0.136 & -.080 & -.117 & .022 & -.041 \\
\hline & $(.116)$ & $(.119)$ & $(.116)$ & $(.121)$ & $(.142)$ & $(.117)$ \\
\hline \multicolumn{7}{|c|}{ CPOE*X } \\
\hline \multirow[t]{2}{*}{ Year 1} & & $-.147 * * *$ & $-.126 *$ & $-.139 * *$ & $-.370 * * *$ & $-.289 * * *$ \\
\hline & & $(.053)$ & $(.067)$ & $(.062)$ & $(.134)$ & $(.252)$ \\
\hline \multirow[t]{2}{*}{ Year 2} & & -.118 & -.097 & -.052 & $-.328 * *$ & $-.378 * * *$ \\
\hline & & $(.086)$ & $(.081)$ & $(.070)$ & $(.151)$ & $(.131)$ \\
\hline \multirow[t]{2}{*}{ Year 3} & & -.028 & -.142 & -.094 & $-.278^{*}$ & $-.289 * *$ \\
\hline & & $(.056)$ & $(.060)$ & $(.068)$ & $(.143)$ & $(.115)$ \\
\hline \multicolumn{7}{|c|}{ F-stat CPOE $+\mathrm{CPOE}^{*} \mathrm{X}=0$} \\
\hline \multicolumn{2}{|l|}{ Year 1} & $3.73 *$ & $2.95^{*}$ & $5.54 * *$ & $5.59 * *$ & $5.78^{* *}$ \\
\hline \multicolumn{2}{|l|}{ Year 2} & $5.06 * *$ & $4.89 * *$ & $5.28 * *$ & $10.15^{* * *}$ & $13.76^{* * *}$ \\
\hline \multicolumn{2}{|l|}{ Year 3} & 1.86 & $3.24 *$ & $3.31 *$ & $3.86^{* *}$ & $5.12 * *$ \\
\hline \multicolumn{2}{|l|}{$X=$} & $\begin{array}{c}\text { Non- } \\
\text { Seniors }\end{array}$ & $\begin{array}{c}\text { Non- } \\
\text { Complex }\end{array}$ & $\begin{array}{c}\text { Common- } \\
\text { Drg }\end{array}$ & $\begin{array}{c}\text { Low- } \\
\text { Mortality }\end{array}$ & $\begin{array}{l}\text { Non- } \\
\text { Severe }\end{array}$ \\
\hline
\end{tabular}

Note: Coefficient estimates are from separate fixed effect linear probability regressions of the aggregate PSIs on dummies indicating adoption of CPOE (including one year, 2 years, and 3 or more years since adoption) and their interactions with different measures for patient complication (X). All regressions control for hospital fixed effects, year fixed effects, patient characteristics, $\mathrm{X}$, a group specific year fixed effects (X*I_year). All standard errors are clustered at the hospital level.

$* * *-\mathrm{p}<.01, * *-\mathrm{p}<.05, *-\mathrm{p}<.10$ 
Table 9: Extended Model

\begin{tabular}{|c|c|c|c|c|c|}
\hline & (1) & (2) & (3) & (4) & (5) \\
\hline \multirow[t]{2}{*}{ CPOE (a1) } & -.036 & -.003 & .006 & .097 & .056 \\
\hline & $(.082)$ & $(.081)$ & $(.087)$ & $(.097)$ & $(.086)$ \\
\hline \multirow[t]{2}{*}{ CPOE_Non-Elderly (a2) } & $-.077 *$ & .022 & -.075 & $.195^{* * *}$ & -0.013 \\
\hline & $(.041)$ & $(.042)$ & $(.046)$ & $(.071)$ & $(.048)$ \\
\hline \multirow[t]{2}{*}{ CPOE_X (a3) } & & $-.092 *$ & $-.114 *$ & $-.303^{* * *}$ & $-.332 * * *$ \\
\hline & & $(.050)$ & $(.062)$ & $(.098)$ & $(.093)$ \\
\hline \multirow[t]{2}{*}{ CPOE_Non-Elderly_X (a4) } & & $-.112^{* *}$ & -0.027 & $-.221 * * *$ & -0.005 \\
\hline & & $(.056)$ & $(.051)$ & $(.073)$ & $(.051)$ \\
\hline Non-Seniors \& $(1-\mathrm{X}): \mathrm{a} 1+\mathrm{a} 2=0$ & 1.84 & 0.06 & 0.63 & $7.03^{* * *}$ & 0.29 \\
\hline Seniors \& X: $\mathrm{a} 1+\mathrm{a} 3=0$ & & 1.06 & 1.71 & $5.01 * *$ & $7.17 * * *$ \\
\hline $\begin{array}{l}\text { Non-Seniors \& X: } \\
\mathrm{a} 1+\mathrm{a} 2+\mathrm{a} 3+\mathrm{a} 4=0\end{array}$ & & $4.18^{* *}$ & $6.59 * *$ & $6.44 * *$ & $8.59 * * *$ \\
\hline$X=$ & & $\begin{array}{c}\text { Non- } \\
\text { Complex }\end{array}$ & $\begin{array}{l}\text { Common- } \\
\text { Drg }\end{array}$ & $\begin{array}{c}\text { Low- } \\
\text { Mortality }\end{array}$ & $\begin{array}{l}\text { Non- } \\
\text { Severe }\end{array}$ \\
\hline
\end{tabular}

Note: Coefficient estimates are from separate fixed effect linear probability regressions of the aggregate PSIs on dummies indicating adoption of CPOE, its interactions with different measures for patient complication (X) and a dummy for non-elderly. All regressions control for hospital fixed effects, year fixed effects, patient characteristics, $X$, a group specific year fixed effects (X*I_year), non_elderly*I_year and non_elderly*X*I_year; All standard errors are clustered at the hospital level.

$* * *-\mathrm{p}<.01, * *-\mathrm{p}<.05, *-\mathrm{p}<.10$ 
Table 10: Alternative Outcomes

\section{(0)}

(1)

(2)

(3)

(4)

(5)

Inpatient
CPOE
CPOE_X

CPOE+CPOE_X=0

.026
$(.028)$

$\begin{array}{ccccc}.036 & .031 & .036 & .029 & .029 \\ (.040) & (.038) & (.032) & (.065) & (.036) \\ -.027 & -.021 & -.013 & -.016 & -.014 \\ (.043) & (.037) & (.037) & (.086) & (.051)\end{array}$

0.07

0.11

0.48

0.11

0.18

\section{Length of Stay (logarithm)}

\begin{tabular}{lcccccc} 
CPOE & -.396 & -.274 & -.061 & .094 & .846 & .650 \\
& $(.379)$ & $(.435)$ & $(-.470)$ & $(.416)$ & $(.689)$ & $(.465)$ \\
CPOE_X & & -.257 & .321 & $-1.459^{* *}$ & - & - \\
& & $(.418)$ & $(.509)$ & $(.612)$ & $(.810)$ & $(.760)$ \\
& & & & & & \\
CPOE+CPOE_X=0 & & 1.53 & 0.44 & $5.25^{* *}$ & $6.36^{* *}$ & $11.78^{* * *}$ \\
X= & & Non- & Non- & Common- & Low- & Non- \\
& & Elderly & Complex & Drg & Mortality & Severe \\
\hline
\end{tabular}

Note: Coefficient estimates are from separate fixed effect linear probability regressions of inpatient mortality and length of stay on dummies indicating adoption of CPOE and its interaction with different measures for patient complication (X). All regressions control for hospital fixed effects, year fixed effects, patient characteristics, $X$, a group specific year fixed effects (X*I_year). All standard errors are clustered at the hospital level.

$* * *-\mathrm{p}<.01, * *-\mathrm{p}<.05, *-\mathrm{p}<.10$ 
Appendix Table 1: Descriptions of Patient Safety Indicators

\begin{tabular}{|c|c|c|}
\hline PSI \# & PSI Name & Description \\
\hline 9 & $\begin{array}{l}\text { Postoperative Hemorrhage or } \\
\text { Hematoma }\end{array}$ & Bleeding or bruising after an operation \\
\hline 10 & $\begin{array}{l}\text { Postoperative Physiological and } \\
\text { Metabolic Derangement }\end{array}$ & $\begin{array}{l}\text { Deficiency in the amount of oxygen reaching } \\
\text { body tissues or other physiological } \\
\text { complications }\end{array}$ \\
\hline 11 & $\begin{array}{l}\text { Postoperative Respiratory } \\
\text { Failure }\end{array}$ & $\begin{array}{l}\text { Conditions that affect breathing function or the } \\
\text { lungs themselves }\end{array}$ \\
\hline 12 & $\begin{array}{l}\text { Postoperative Pulmonary } \\
\text { Embolism or Deep Vein } \\
\text { Thrombosis }\end{array}$ & $\begin{array}{l}\text { Blockage of main artery of the lung or one of } \\
\text { its branches by a substance that has travelled } \\
\text { from elsewhere in the body or a blood clot in a } \\
\text { deep vein }\end{array}$ \\
\hline 6 & Iatrogenic Pneumothorax Rate & $\begin{array}{l}\text { a condition in which air or gas is present in the } \\
\text { pleural cavity as a result of mechanical } \\
\text { ventilation, tracheostomy tube placement, or } \\
\text { other therapeutic intervention }\end{array}$ \\
\hline 7 & $\begin{array}{l}\text { Central Venous Catheter-Related } \\
\text { Blood Stream Infection }\end{array}$ & $\begin{array}{l}\text { Blood infection arising from the tubular, } \\
\text { flexible surgical instrument that is inserted into } \\
\text { a cavity of the body to withdraw or introduce } \\
\text { fluid. }\end{array}$ \\
\hline 8 & Postoperative Hip Fracture Rate & $\begin{array}{l}\text { Hip fractures after an operation when hip } \\
\text { fracture was not present on admission } \\
\text { excluding some cases such as muscular } \\
\text { disorders or violent seizures where fracture } \\
\text { may not be fault of hospital }\end{array}$ \\
\hline 15 & $\begin{array}{l}\text { Accidental Puncture or } \\
\text { Laceration Rate }\end{array}$ & Accidental puncture or cut during procedure \\
\hline
\end{tabular}

